\title{
BIOPROT Contenedor Autónomo de Residuos Biológicos.
}

\section{BIOPROT Autonomous Container of Biological Residues.}

\author{
Brodny García Márquez, Jhoel Castro Amaya. \\ Universidad Francisco de Paula Santander Ocaña, \\ Facultad de ingenierías, ingeniería de sistemas, Ocaña, Norte de Santander, Colombia \\ +573157045858bagarciam@ufpso.edu.co, +573182981401 jcastroa@ufpso.edu.co
}

\begin{abstract}
Resumen: Los desechos biológicos generados en los centros de salud son una gran fuente de microorganismos como bacterias, parásitos, virus y hongos con el suficiente grado de concentración como para producir enfermedades infecciosas, lo cual hace indispensable que el personal que labora en dichos centros, cuente con mecanismos de protección que minimicen el riesgo de contraerlos.

El objetivo principal es evitar que el personal entre en contacto con microorganismos que pueden provocar infecciones debido al mal manejo de los residuos biológicos, al desarrollar una herramienta que de manera autónoma realice actividades que de hacerlas un ser humano pueden afectar su integridad física; aplicando las bondades de la tecnología al usar dispositivos electrónicos y herramientas tecnológicas. Además, proponer opciones ventajosas para el correcto manejo de estos residuos, en los diferentes establecimientos de salud.
\end{abstract}

Palabras clave: Residuos biológicos, bioseguridad, contenedor inteligente, prevención, riesgos laborales.

\begin{abstract}
Biological waste generated in health centers is a great source of microorganisms such as bacteria, parasites, viruses and fungi with a sufficient degree of concentration to produce infectious diseases, which makes it essential that the personnel working in these centers, have protection mechanisms that minimize the risk of contracting them.

The main objective is to prevent personnel from coming into contact with microorganisms that can cause infections due to the mishandling of biological residues, by developing a tool that autonomously carries out activities that, in order to make them a human being, can affect their physical integrity; Applying the benefits of technology when using electronic devices and technological tools. In addition, the advantageous options for the correct handling of this waste, in the different sectors of health.
\end{abstract}

Keywords: Biological waste, biosecurity, intelligent container, prevention, occupational hazards.

\section{INTRODUCCIÓN}

En la actualidad, entre las enfermedades infecciosas a las que están expuestos los profesionales de salud, que presentan un accidente por riesgo biológico, se destacan aquellas de etiología vírica como la Hepatitis B, Hepatitis C,
Hepatitis Delta y el SIDA, sin olvidar otros virus y enfermedades producidas por otros microorganismos (tétanos, TBC, legionelosis, fiebre Q, rubéola). (Ministerio de vivienda, 2015)

Los residuos contienen mercurio y otros metales pesados, solventes químicos y conservantes (ej, 
formaldehído), los cuales se sabe que son cancerígenos, y plásticos (ej. PVC), que cuando se quema produce dioxinas y otros contaminantes que acarrea serios riesgos para la salud del hombre; no sólo para los trabajadores, sino también para el público en general a través del suministro de alimentos. (Shaner, 1997) (G Sandoval, JT Molano. 2013).

Así, que es de vital importancia el fomentar la toma de conciencia en los trabajadores de la salud, con respecto a los actos, condiciones de trabajo y la ejecución de las medidas preventivas, contribuyendo así a controlar la accidentalidad y por tanto promover la calidad de vida de los profesionales de la salud. Se debe fomentar la participación activamente en la elaboración de Manuales de procedimientos y actos seguros. Todo aporte es de gran ayuda para los Administradores de Riesgos Profesionales y para las áreas de Salud Ocupacional, quienes deben participar integralmente y en forma personalizada en la sensibilización, seguimiento y control de las prácticas y condiciones seguras de trabajo en el ambiente hospitalario, además de dar a conocer la situación actual de la accidentalidad por riesgo biológico en el personal sanitario, esta contribuye al diseño de protocolos de prevención de riesgo biológico, manejo de residuos y desechos hospitalarios, manuales para estandarización de procesos adecuados y seguros, cursos de entrenamiento, capacitación y formación del personal de la salud. (Ministerio de vivienda, 2015)

El objetivo primordial de todos los mecanismos implementados en manejar correctamente los residuos biológicos en los centros de salud, así como proteger el medio ambiente y a la población que pudiera estar en contacto con estos residuos dentro de las instituciones de atención médica. (who.com, 2017)

Para evitar que los RPBI (Residuos Peligrosos Biológico-Infecciosos) se mezclen con la basura común, se debe preestablecer un sitio para su almacenamiento temporal. Los RPBI deberán almacenarse en contenedores con tapa y permanecer cerrados todo el tiempo. No debe de haber residuos tirados en los alrededores de los contenedores. (Trujillo y Vides, 2008) (LAM Mesa, NB Lombana. 2013)

Todos los mecanismos y normas empleados son importantes para el control de la seguridad laboral en centros hospitalarios, entre ellos se encuentra el PGIRS, que es el instrumento de planeación municipal o regional que contiene un conjunto ordenado de objetivos, metas, programas, proyectos, actividades y recursos definidos por uno o más entes territoriales para el manejo de los residuos sólidos, fundamentado en la política de gestión integral de los mismos, el cual se ejecutará durante un período determinado, basándose en un diagnóstico inicial, en su proyección hacia el futuro $\mathrm{y}$ en un plan financiero viable que permita garantizar el mejoramiento continuo del manejo de residuos sólidos y la prestación del servicio de aseo a nivel municipal o regional, evaluado a través de la medición permanente de resultados. (Decreto 1077 de 2015 expedido por el Ministerio de Vivienda, Ciudad y Territorio). (Santos y Rivero, 2003)

\section{SOLUCIÓN PLANTEADA}

Desarrollar una herramienta que de manera autónoma realice actividades que de hacerlas un ser humano pueden afectar su integridad física; aplicando las bondades de la tecnología al usar dispositivos electrónicos y herramientas tecnológicas, además sugerir alternativas útiles para el manejo de estos residuos, en los establecimientos de salud.

Estas inseguridades laborales pueden ser minimizadas al desarrollarse un artefacto tecnológico manipulado por medio de un aplicativo móvil, logrando que esta actividad se pueda realizar de forma higiénica, segura y sin esfuerzo.

El mecanismo realiza una serie de actividades comenzando por la recolección de los residuos en un contenedor, el cual detecta cuando el empleado desea ingresar los desechos abriendo su tapa sin necesidad de contacto con los otros residuos allí depositados. Una vez el contenedor reconoce por medio de un sensor que se encuentra al $80 \%$ de su capacidad, envía un mensaje al dispositivo móvil del empleado, solicitando autorización para proceder a sellar la bolsa aplicando calor a esta. Posteriormente, el contenedor se dispone a trasladar la bolsa ya sellada a un lugar previamente asignado y luego retornar a su lugar de origen e iniciar de nuevo el proceso.

\section{METODOLOGÍA}

Para el desarrollo del contenedor autónomo de residuos biológicos primero se realizó una investigación en centros de salud, para conocer de primera mano las necesidades de higiene y protección a empleados en cuanto al manejo de residuos biológicos en la entidad. 
Para esto se contó con la asesoría de profesionales como:

- $\quad$ Yaqueline Pabón Bacca: Administradora de Empresas, Esp. Gerencia Riesgos Laborales Seguridad y Salud en el Trabajo Clínica Nuestra Señora de Torcoroma.

- $\quad$ Felisa Beatriz Carvajalino Calle: Medica Patóloga, C.I.S Laboratorio Clínico Especializado, Médico UFPSO.

- Claudia Patricia Ahumada Mozo: Odontóloga Odontosonrie.

Para los siguientes pasos de la elaboración del contenedor, se establecieron cuatro objetivos principales, los cuales se desarrollaron en cinco tiempos consecutivos los cuales fueron:

- $\quad$ Construir una caneca que por medio de un sensor de distancia detecte cuando se desea ingresar basura al contenedor y esta abra su tapa sin necesidad de tocarla.

- Implantar en el contenedor un sensor de distancia que detecte cuando se encuentre llena y lo comunique a un dispositivo móvil.

- $\quad$ Elaborar un mecanismo para que la caneca selle la bolsa de basura cuando el usuario lo ordene por medio de la aplicación móvil.

- Realizar un sistema móvil que siga determinado recorrido de forma autónoma, guiados por sensores o rutas que han sido cargadas o enviadas mediante una aplicación móvil.

El primer objetivo se logró realizar inicialmente con un mecanismo que funcionaba por medio de un solenoide, el cual hacía abrir la tapa de manera vertical. Este sistema funcionó en las dos primeras etapas, dando lugar al primer prototipo funcional, el cual se muestra más adelante. Posteriormente se modificó por razones de practicidad e higiene, por un sistema basado en una unidad de CD.

Inicialmente el primer objetivo se logró realizar con los siguientes materiales:

Sensor de proximidad, solenoide, arduino nano, relay, transformador, caneca de plástico

Su mecanismo estaba basado en la utilización de un solenoide que es el que realiza el movimiento de la tapa, pues éste es un componente electrónico que usa una bobina de alambre de cobre en la cual, al energizarse, crea un campo magnético actuando como un imán. En la mitad va un hierro que es la varilla que sube y baja que al haber un campo magnético éste es atraído o repelado. En nuestro caso era repelado y por ésta razón se abría la tapa.

Para el segundo objetivo, se buscaba saber cuál era el nivel de residuos en el contenedor, para esto se utilizaron los siguientes materiales:

Sensor de proximidad, arduino nano, módulo bluetooth HC-05

Un sensor de proximidad es un transductor que detecta objetos o señales que se encuentran cerca del elemento sensor, y un módulo bluetooth hc-05 es un módulo que se puede utilizar en todo tipo de proyectos donde se necesite una conexión inalámbrica fiable y sencilla de utilizar. Se configura mediante comandos AT y tiene la posibilidad de hacerlo funcionar tanto en modo maestro como esclavo.

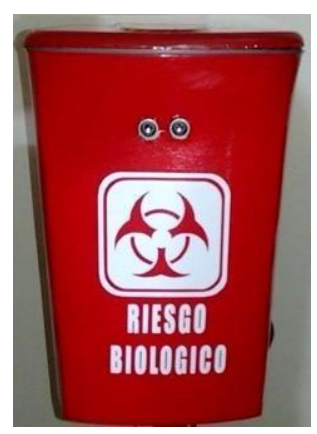

Fig. 1. Primer prototipo funcional llevado a cabo en los dos primeros tiempos del proyecto.

Antes de iniciar el tercer objetivo, se vio la necesidad de cambiar el diseño, materiales y ubicación de los dispositivos en el contenedor. Los cambios fueron los siguientes:

- $\quad$ Se cambió el material inicial (plástico) por un material más resistente y estable llamado melamina, haciendo del contenedor un producto más seguro y de mejor calidad.

- Se cambió la ubicación del sensor de distancia, que permitía abrir la tapa, para que fuera más cómodo el ingreso de los desechos y a su vez evitar que acciones involuntarias hicieran abrir la tapa innecesariamente. 
- $\quad$ Se cambió la estructura que permitía abrir la tapa de manera vertical (basado en un solenoide), por un sistema basado en una unidad de $\mathrm{CD}$ que la abre de manera horizontal, evitando accidentes con los residuos y mejorando el diseño del contenedor.

Para el tercer objetivo, se requería sellar la bolsa una vez estuviera llena, esto se logró utilizando partes de una selladora de bolsas electrónica y una unidad de cd en donde las resistencias de la selladora fueron adheridas al sistema de la unidad logrando un perfecto acoplamiento en el contenedor.

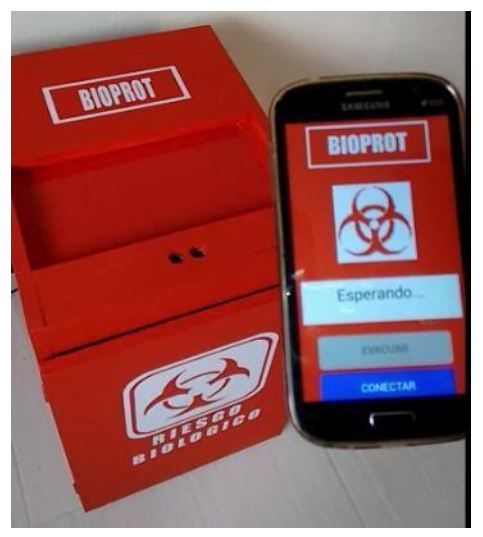

Fig. 2. Segundo prototipo funcional en el tiempo cuatro del proyecto.

Para el cuarto objetivo, se desea que el contenedor transporte la bolsa ya sellada hacia un lugar preestablecido por medio de un seguidor de línea, cuyo objetivo es seguir una línea marcada en el suelo de color negro sobre un piso blanco, propio de centros de salud.

El desarrollo de robots móviles responde a la necesidad de incrementar la autonomía para limitar todo lo posible la intervención humana. La autonomía de un robot móvil se basa en el sistema de navegación automática, que cuenta con sensores infrarrojos que detectan la línea, y mediante un dispositivo digital de control, realizan una acción de revisión establecida en un programa que dirige al robot en una secuencia de órdenes para que este mantenga la trayectoria demarcada. (Calderón et al., 2017)

\section{ANÁLISIS DE RESULTADOS Y DISCUSIÓN}

Se concluyó que en la mayoría de estos centros cuentan con contenedores para este tipo de desecho, pero que reconocen sus limitaciones en cuanto a protección al trabajador, pues se notaron varios puntos débiles en el actual sistema recolector:

- $\quad$ En algunos centros se notó que los niveles de residuos en los contenedores excedían el límite legal de $80 \%$ de la capacidad de este.

- $\quad$ Se pudo apreciar la dificultad en el cumplimiento del vaciado de la caneca por parte de personal encargado e imposible por parte de médicos, bacteriólogos y demás profesionales de la salud, puesto que extralimitan sus funciones.

- $\quad$ Presentan un difícil manejo de otro tipo de residuos biológicos distintos a los estudiados, como material corto-punzante y material de tejido humano con consistencia líquida.

Después de haber llevado a cabo los objetivos establecidos, el Contenedor Autónomo para Residuos Biológicos BIOPROT, está compuesto por varios dispositivos electrónicos como lo son dos sensores de proximidad, con los cuales el contenedor detecta cuando desea ingresar un elemento a la caneca, y otro que nos indica el nivel de residuos que contiene en tiempo real a través de un dispositivo móvil.

Este mecanismo evita que el empleado toque la caneca para introducir elementos, así como también controlar que no excedan el límite establecido por la ley que es del $80 \%$ del contenedor y que para los encargados de la recolección es de difícil cumplimiento y no es función de médicos, bacteriólogos y demás profesionales de la salud. (Ministerio de vivienda, 2015)

Una vez se llevó el contenedor BIOPROT a los centros de salud, se tuvo la posibilidad de recibir recomendaciones para prototipos futuros $\mathrm{y}$ continuar con el mejoramiento y optimización del sistema, de los cuales podemos sintetizar en los siguientes:

- La abertura proporcionada para la inserción de los desechos podría haber sido más amplia y así aumentar la comodidad del empleado.

- $\quad$ Aunque el equipo de trabajo de BIOPROT basó su segundo prototipo en aras de implementar un mecanismo lavable, esto no se pudo llevar a 
cabo pues no hacía parte de los objetivos principales de esta etapa, sin embargo, los profesionales de la salud que analizaron el contenedor también hicieron énfasis en la importancia de esta característica en futuros prototipos.

- $\quad$ Se pretende que el contenedor sea completamente autónomo, por lo tanto, los profesionales recomiendan que se encuentre la forma de que el empleado no sea quien reemplace la bolsa, sino que el sistema lo haga por sí mismo.

\section{CONCLUSIONES}

Podríamos decir que no importa qué estrategia final se adopte para el tratamiento y disposición final de los residuos, es fundamental que los residuos se clasifiquen (preferentemente en el lugar en el que se generan) previamente a tratarlos y disponerlos. Este paso tan importante debe ser dado para salvaguardar la salud laboral del personal del establecimiento.

Para el desarrollo de este aparato se tomaron en cuenta algunas situaciones que son poco higiénicas y que pueden afectar severamente la salud tanto del paciente como del personal encargado de realizar la recolección, por ello se implementó un mecanismo que por medio de sensores y otros artefactos pueda, primero, abrir la tapa, segundo, mandar un mensaje a una aplicación móvil indicando el nivel de la basura, tercero, que cuando esta se encuentre llena el usuario sea quien decida si quiere que se cierre la basura y por último, que cuando se le indique, el contenedor transporte la basura por medio de una base con ruedas utilizando el sistema de un seguidor de línea, para su traslado al sitio deseado.

Por último, cabe resaltar que BIOPROT es un proyecto que se inició en el curso de Electrónica General y se le dio continuidad en Circuitos Lógicos, pero que requiere de un trabajo adicional en un semillero de investigación donde se podrán hacer las adiciones pertinentes y donde se tomarán en cuenta todas las sugerencias de los profesionales de la salud para obtener un producto con posibilidades reales en el mercado.

\section{RECONOCIMIENTO}

Para la elaboración de este proyecto fue indispensable la colaboración de profesionales que nos brindaron un aporte valioso al desarrollo de este proyecto como lo son: la especialista en gerencia de riesgos laborales de seguridad y salud en el trabajo, Yaqueline Pabón Bacca; la médica patóloga, Felisa Beatriz Carvajalino Calle; la odontóloga, Claudia Patricia Ahumada Mozo. Así como también se hace una especial mención a la ingeniera electrónica, Diana Marcela Rodríguez Arciniegas y a la universidad Francisco de Paula Santander Ocaña por el apoyo brindado.

\section{REFERENCIAS}

C. y. T. d. C. Ministerio de Vivienda, «Ministerio de Vivienda, Ciudad y Territorio de Colombia,» 2015. http://www.minvivienda.gov.co/viceminis terios/viceministerio-de-agua/planes-degestion integral-de-residuos-solidos.

G Sandoval, JT Molano. (2013), Pluviógrafo electrónico con transmisión de datos inalámbrica. Revista Colombiana de Tecnologías de Avanzada ISSN: 16927257.

L. A. M. Mesa, N. B. Lombana. (2013). La robótica educativa como instrumento didáctico alternativo en educación básica. REVISTA COLOMBIANA DE TECNOLOGÍAS DE AVANZADA, ISSN: 1692-7257. 2(22).

L. Mesa Mesa and N. Barrera Lombana. (2013). LA ROBÓTICA EDUCATIVA COMO INSTRUMENTO DIDÁCTICO ALTERNATIVO EN EDUCACIÓN BÁSICA. REVISTA COLOMBIANA DE TECNOLOGÍAS DE AVANZADA, vol. 2, no. 22.

O. Trujillo Polanía y A. Vides San Juan, «Universidad Javeriana,»15 Enero 2008. http://javeriana.edu.co/biblos/tesis/enferm eria/tesis34.pdf.

C. Santos Buroga, L. Rivero Rodriguez y L. Rodriguez Cabrera, Noviembre 2003. http://www.promocion.salud.gob.mx/dgps /descargas1/influenza/mat/Guia_manejo_d e_residuos_biologicos.pdf.

J. Guzmán-Luna, I. D. Torres, J. F. Alvarez. (2014). Propuesta de un generador de aplicaciones educativas basadas en televisión digital usando arquitectura de cómputo en la nube. REVISTA COLOMBIANA DE TECNOLOGÍAS DE AVANZADA, ISSN: 1692-7257. 1(23).

J. Guzmán Luna, I. Torres and J. Alvarez. (2014). Propuesta de un generador de aplicaciones educativas basadas en televisión digital usando arquitectura de cómputo en la nube. REVISTA COLOMBIANA DE TECNOLOGÍAS DE AVANZADA, vol. 1, no. 23, 2014.

N. y. G. M. Hollie Shaner, «11 Recomendaciones para,» CGH Environmental Strategies, 
1997.

O. M. d. 1. Salud, «who.com,» http://www.who.int/es/

Calderón, H. A., Mejía, C., \& Cobo, L. (2017). Implementación de un robot móvil seguidor de línea y detector de obstáculos con comunicación Bluetooth. Revista Ontare, 4(2). 